\title{
Identifying Critical Clusters of Traffic-Loading Events in Recurrent Congested Conditions on a Long-Span Road Bridge
}

\author{
E. Alexandra Micu ${ }^{1} * \mathbb{C}^{\mathbb{D}}$, Eugene J. OBrien $\left.{ }^{1}{ }^{(}\right)$, Abdollah Malekjafarian ${ }^{1}{ }^{\circledR}$, Ross McKinstray $^{2}$, \\ Ewan Angus ${ }^{2}$, Myra Lydon ${ }^{3}$ and F. Necati Catbas 4 (iD) \\ 1 School of Civil Engineering, University College Dublin, D04 V1W8 Dublin, Ireland; \\ eugene.obrien@ucd.ie (E.J.O.); abdollah.malekjafarian@ucd.ie (A.M.) \\ 2 Amey Consulting, Forth Road Bridge, Administration Building, South Queensferry EH30 9QZ, UK; \\ Ross.Mckinstray@amey.co.uk (R.M.); Ewan.Angus@amey.co.uk (E.A.) \\ 3 School of Natural and Built Environment, Queen's University Belfast, David Keir Building, \\ Belfast BT9 5AG, UK; M.Lydon@qub.ac.uk \\ 4 Civil Infrastructure Technologies for Resilience and Safety, University of Central Florida, \\ Orlando, FL 32816-2450, USA; catbas@ucf.edu \\ * Correspondence: alexandra.micu@ucdconnect.ie; Tel.: +353-1-7163231
}

Received: 10 July 2020; Accepted: 3 August 2020; Published: 5 August 2020

\begin{abstract}
This paper examines the nature of traffic loading in recurrent congested traffic conditions on a long-span suspension bridge. Traffic flow and percentage of trucks are extracted from image data and a cluster analysis performed to classify the data into four clusters. One cluster (MTHF, medium truck percentage and high flow) is identified that incorporates almost $50 \%$ of the hours of traffic data scattered throughout the day. Site-specific load assessment confirms that this MTHF cluster is the most critical for the bridge considered, the Forth Road Bridge in Scotland. For non-recurrent congestion, another cluster (HTLF, high percentage of trucks and low flow) is shown to govern but this finding is highly site-specific, depending on the relative frequency of the different types of congestion. A comparison of the maximum hourly/daily MTHF load effect of the cable force for five notional bridges shows that a $100 \%$ increase in the bridge span generates an increase of about $65 \%$ in the characteristic load effect.
\end{abstract}

Keywords: bridge; cluster; congestion; dendrogram; flow; image; loading; long-span; non-recurrent; recurrent; suspension; traffic

\section{Introduction}

Traffic loading represents a key component in the safety assessment of an existing bridge [1,2]. For short- and medium-span bridges, free-flowing traffic with an allowance for dynamic interaction, is the critical loading condition. This typically involves no more than one or two trucks on the bridge at the same time. On the other hand, congested traffic with multiple closely spaced vehicles, governs for longer spans [3-6]. Furthermore, as would be expected, critical congested situations involve the simultaneous presence of large numbers of trucks on the bridge.

From a long-span bridge loading perspective, the car/truck mix and inter-vehicle gaps are key properties of congested traffic. Worldwide, traffic data are gathered by weigh-in-motion (WIM) technologies, typically consisting of piezo-electric sensors embedded in the road pavement $[7,8]$. While these technologies work well in free-flowing traffic, they are ineffective in slow moving and/or stop-and-go traffic [9], i.e., for those conditions which are critical for long span bridges. Therefore, vehicle weight data are not readily available on the bridge, with associated congested 
car/truck mixes and inter-vehicle gap statistics. In this study, a camera mounted on a tower of the Forth Road Bridge (FRB) in Scotland is used to estimate the lengths of vehicles and to gather data on inter-vehicle gaps and the car-truck mix. Data from a WIM station nearby is used to establish the correlation between length and weight, and this is used to estimate the weights of vehicles on the bridge.

Traffic loading on the bridge is investigated for conditions of recurrent congestion, i.e., congestion that occurs on a regular (daily) basis (as opposed to non-recurrent congestion due to, for example, an accident). Load effects are investigated for an extensive range of flows and traffic compositions, involving two thousand hours of video data recorded over five months. Data in the images is clustered into four homogenous groups based on the overall traffic flow and the truck percentage. The site-specific characteristic maximum load is evaluated for the most critical cluster for the FRB and for four notional bridges.

The aim of much traffic loading research is to propose an appropriate model for long-span bridges. Given the problems with collecting congested traffic data, most work to date is based on available free-flowing data [10-16]. Many researchers create congestion by 'collapsing' free-flowing traffic, i.e., by reducing the recorded inter-vehicle gaps (axle-to-axle or bumper-to-bumper) to minimum values [5,15-20]. This is inappropriate for multi-lane traffic as it changes the relative positions of vehicles in adjacent lanes. This removes overtaking events from the data in which lighter vehicles are overtaking heavier ones. Overtaking events cause subtle correlations in the weights of traffic in adjacent lanes, correlations that are lost if inter-vehicle gaps are collapsed.

Microsimulation has been used to address the interaction between vehicles for multi-lane traffic as it allows for vehicle lane changes as traffic becomes congested [6,13,21,22]. Microsimulation [23] is based on the assumption that every single vehicle is characterized by unique parameters of driver behaviour. Traffic composition and vehicle interaction is studied in various traffic conditions by OBrien et al. [14] using microsimulation software. OBrien et al. [9] simulate congestion on a single lane long-span bridge using in-house microsimulation software. They conclude that different forms of congestion for traffic with different percentages of trucks can lead to the bridge being critically loaded, but there is no dominant one. Caprani, et al. [24] implement lane changing and car-following models for multi-lane traffic loading, the two key elements of a microsimulation model. However, most of the aforementioned congested traffic loading models are developed on the basis of free-flowing traffic records (vehicle weights and traffic composition). Congested traffic data are still necessary to calibrate and validate these models [25].

The potential of computer vision and image-processing techniques has been exploited for traffic surveillance for many years [26]. Image/video traffic data are analysed to detect $[27,28]$ and classify the vehicles $[29,30]$. Information can also be found in image/video data on traffic density [31], vehicle speed [32,33] and driver behaviour [34] for different conditions of weather and traffic. For bridge loading, Nowak, et al. [19] use video data to analyse the distribution of trucks on multi-lane bridges in congested traffic. The composition of traffic on the roads of the United Kingdom is investigated by Ricketts and Page [35] using video data. A microsimulation model is calibrated by OBrien et al. [14] based on traffic composition and driver behaviour for different traffic conditions observed in video data. Micu, et al. [36] explore the potential of cameras and image processing techniques to collect congested traffic data. The feasibility is established of extracting valuable information from images such as traffic composition, vehicle lengths and bridge load effects.

\section{Forth Road Bridge Vehicle Length Data}

The Forth Road Bridge (Figure 1) is a $2.5 \mathrm{~km}$ suspension bridge over the River Forth, North of Edinburgh, Scotland. In September 2017, a new bridge was opened upstream of the site and FRB was restricted to public service vehicles. Before that closure, general traffic data was collected on the FRB by a video system and nearby using WIM technology. This pre-September 2017 data is investigated in this paper. The data analysis methods are summarized in this section and explained in detail in [36,37]. 


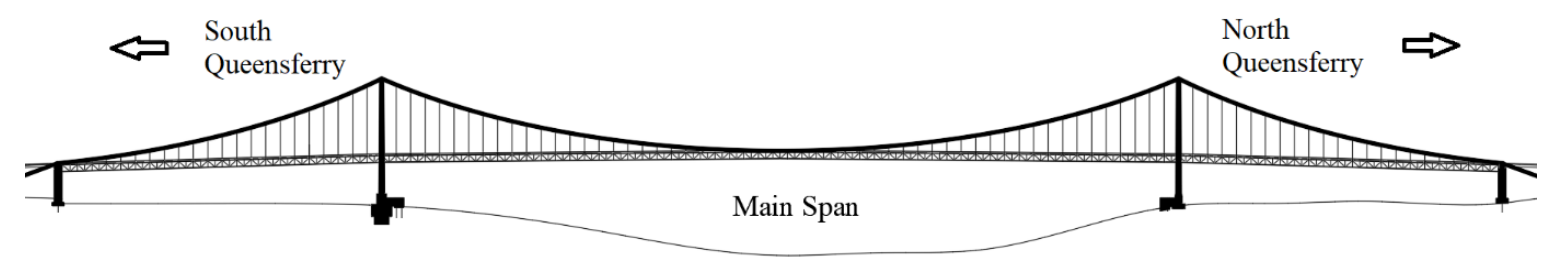

Figure 1. Longitudinal elevation of the Forth Road Bridge (FRB).

\subsection{Vehicle Lengths in Traffic Image Data}

A video system was installed on the south main tower to survey the traffic on the FRB. The video system includes an Internet protocol camera, a network video recorder (NVR) and a power-over-ethernet (PoE) unit. The camera has pan/tilt/zoom (PTZ) capability and is suitable for outdoor use. Continuous serviceability of the camera was ensured by the infrared-cut filter. Electric power and data transfer were supplied by the PoE unit using a single cable. The NVR provides access to settings of the camera and data records.

The system collected video traffic data at a rate of 1 frame per second from April to August 2017, before the traffic was redirected to the new bridge. Only daytime traffic from 5 am to $10 \mathrm{pm}$ on the northbound carriageway is considered in this research. The night-time traffic data were removed from the database due to a large number of images of low quality and traffic with low flow. A further group of 203 out of $2210 \mathrm{~h}$ of video data were removed due to challenging weather conditions (wind, sun or fog). Videos were segmented into frames and each frame analysed separately.

A digital image can be represented numerically as three matrices, one for each of the primary colours. Adaptive thresholding [38] and morphological reconstruction [39] are applied to analyse the frames and extract the key traffic features such as vehicle outline. The outline of each vehicle is then enclosed in a rectangle and its location and dimensions extracted. Details of the image processing method are given in $[37,40]$. Approximately 3 million northbound vehicles, representing $95 \%$ of the data in these images, were identified in 130 days of records. Figure 2 gives an example of an image being analysed step by step and vehicle length being extracted.

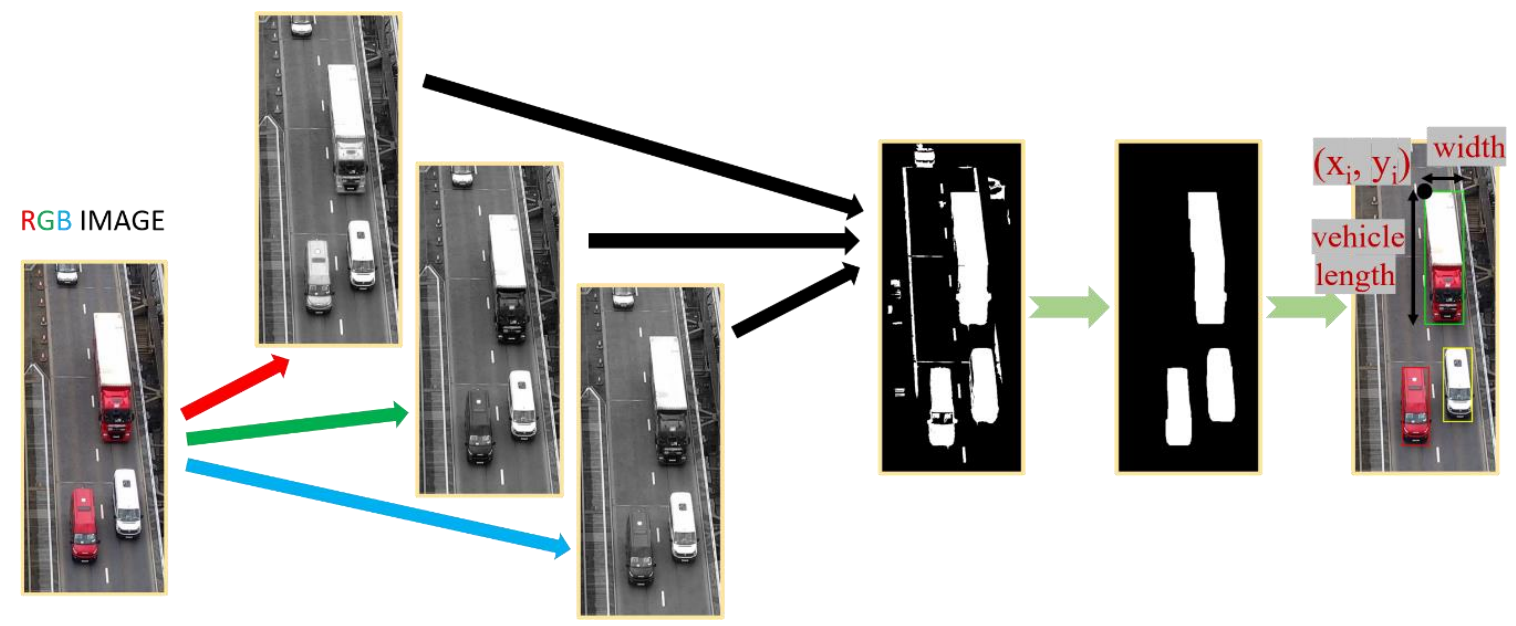

Figure 2. Vehicle length measured in images: primary intensities (red, green, blue) extracted from colour image and represented as individual grey images; results of red, green and blue intensities applied together; vehicle outlines identified and enclosed in rectangles [41].

One year of WIM records are available from a WIM system at the South end of the FRB. Different data cleaning criteria were applied [42] to ensure a clean set of data. The cleaned database of free-flowing vehicles was used to establish the statistical correlation between vehicle length and weight. Figure 3a provides a bivariate histogram of length and weight data for one month of slow-lane traffic, 
i.e., the colours indicate the frequency of each length/weight combination. This can be used to extract a gross weight histogram for vehicles of any given length. Here the histogram is shown for vehicles in the length range of 9.9 to $10.2 \mathrm{~m}$ (Figure 3b). Having found the histogram, the probability density function (PDF) is estimated using a univariate kernel density estimator (KDE) [43]—Figure 3c. Hence the cumulative distribution function (CDF) is found-Figure 3d. Having established the CDF, Monte Carlo sampling is used to infer a 'typical' weight for the vehicle recorded in the image. While this process is clearly inaccurate for individual vehicles, it has been shown to be quite accurate in probabilistic calculations of the type considered here [36].

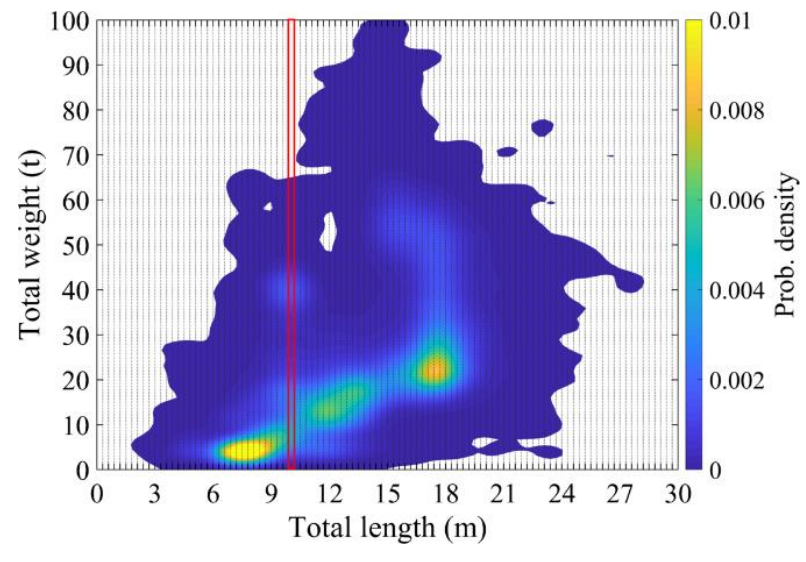

(a)

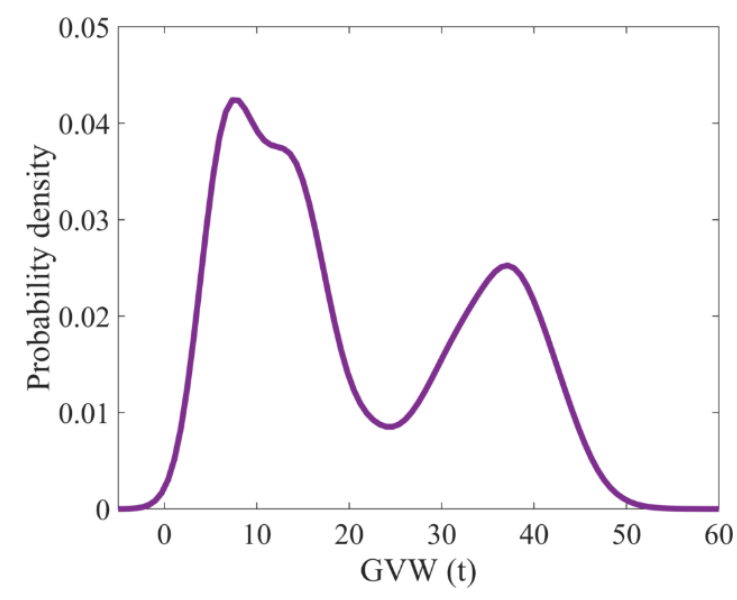

(c)

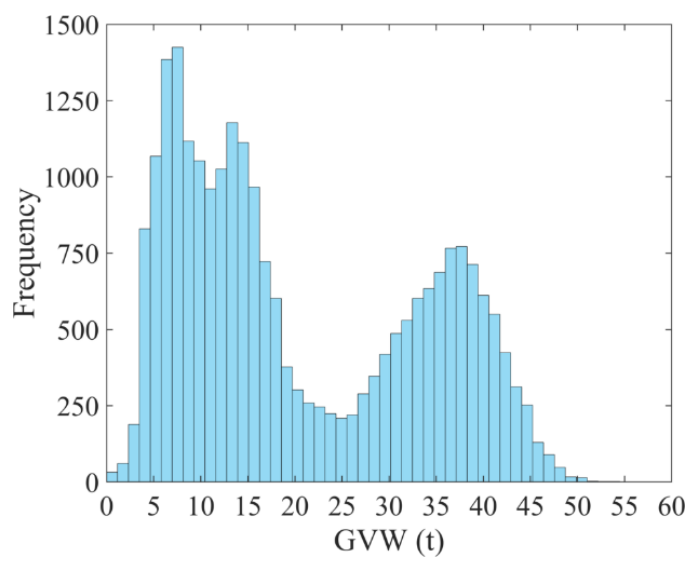

(b)

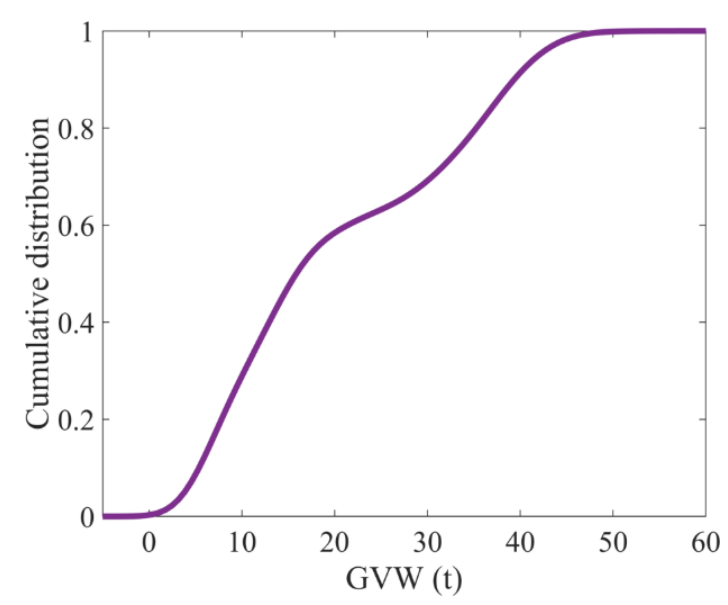

(d)

Figure 3. Weight inference process illustrated with one month of slow lane vehicle weights: (a) Contours of relative frequency of weight/length combinations; (b) Gross weight (GVW) histogram for segment 34 (9.90-10.20 m); (c) Fitted probability density function; (d) Cumulative distribution function.

\section{Cluster Analysis for Data Classification}

It is well known in traffic theory that flow and density of traffic are linearly related until congestion occurs and the relationship breaks down. At this point, mean traffic speed slows, resulting in reduced flow. From a bridge loading perspective, the density of traffic determines the number of vehicles on the bridge. Truck percentage is also key as trucks are much heavier than cars. Figure 4 illustrates the data extracted from images in terms of traffic flow (vehicles per hour) and the average percentage of trucks (per hour). In the hours when flow is highest, the \% trucks tends to be less, and the really high truck percentages are when flow is less than about $500 \mathrm{veh} / \mathrm{h}$-clearly many truck drivers choose to 
make the journey when traffic flow is low. Cluster analysis [44,45] is used here to separate the data into a number of clusters whose implications for bridge loading can be considered separately. In cluster analysis, similarities between objects are measured as distances between points on the graph [46]. Thus, a cluster is a group of points that are relatively close to each other and are distinctly less close to points in other clusters.

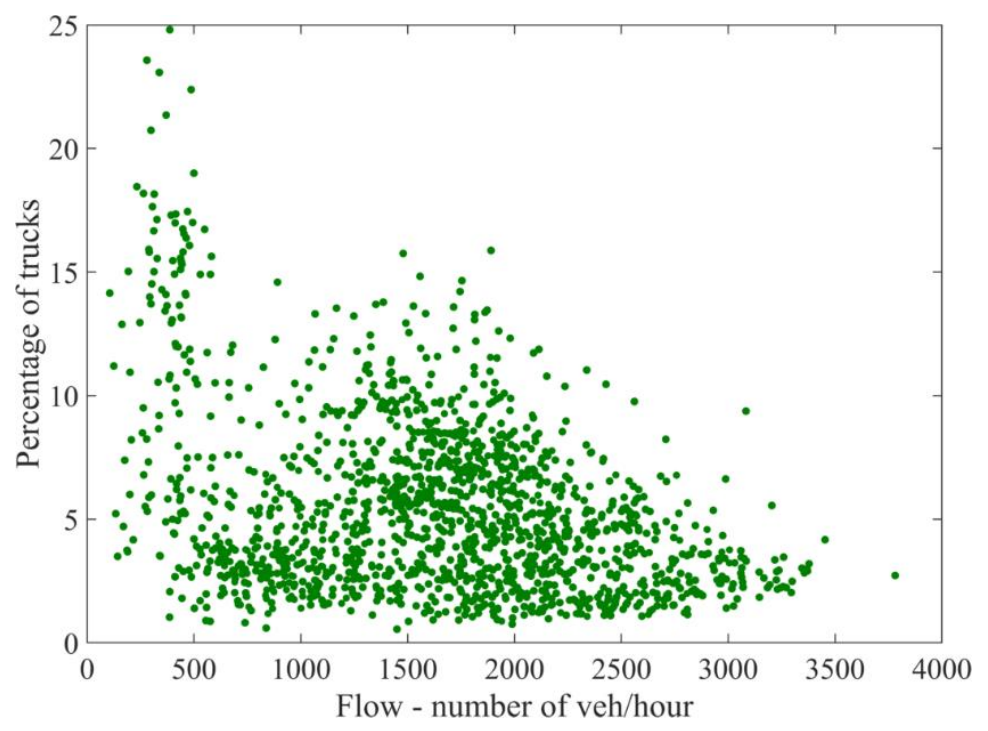

Figure 4. Correlation between traffic flow and percentage of trucks.

While graphical methods can provide evidence of cluster structure in data, they tend to be subjective [47]. A hierarchical approach, consisting of a series of data segmentations, provides an objective approach. This method involves progressive data subdivision or synthesis at each stage of segmentation [48]. The hierarchical classification is often represented by a tree structure referred to as a dendrogram [49] which shows the clustering structure at each stage-Figure 5. In a 'bottom up' approach, it starts by assuming that each point is an individual cluster. At each stage, the two most similar clusters generate a new cluster. Each object is attributed to one cluster only.

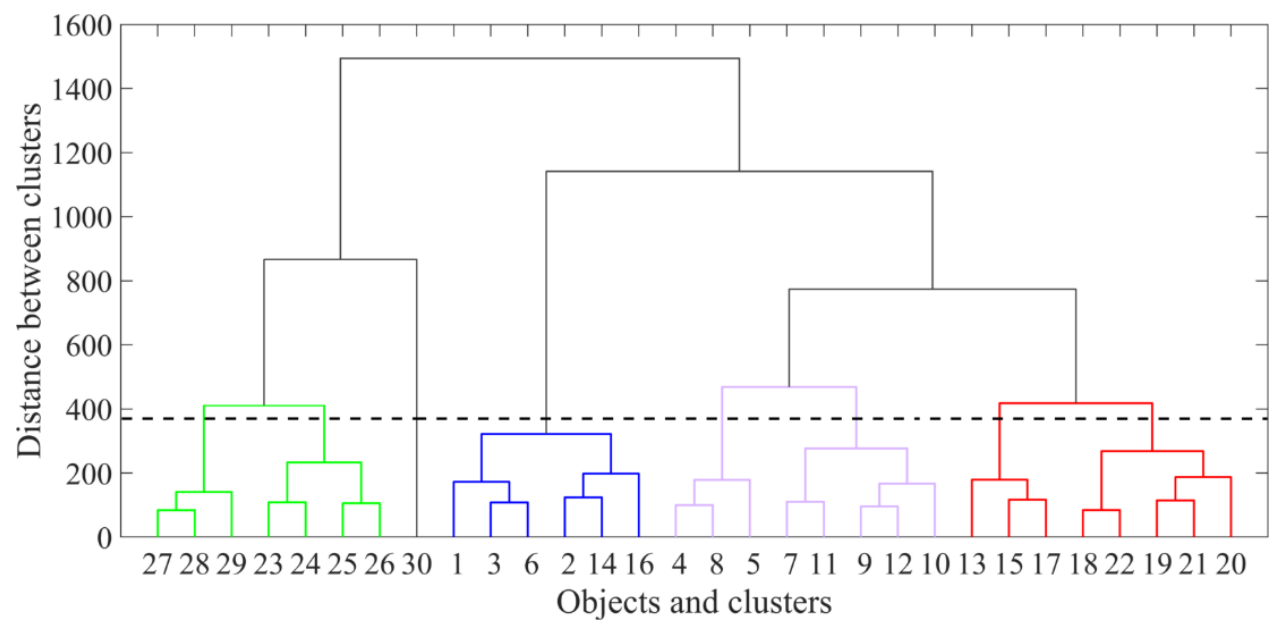

Figure 5. Dendrogram: the traffic flow-percentage of trucks pairs attributed to correct/natural/optimal clusters.

The dendrogram of Figure 5 shows the objects (vehicle flow/truck density) on the $\mathrm{X}$-axis and the distances (or differences) between clusters on the Y-axis. The horizontal lines in the dendrogram represent the clusters while the vertical lines are the distances that clusters are connected 
at. The correct/natural/optimum number of clusters is indicated by horizontal lines positioned at approximate the same level on the Y-axis. The black dashed line in Figure 5 shows there are four correct/natural/optimal clusters in this dataset. Each cluster is highlighted in this figure by a different colour.

Figure 6 shows the four clusters on the scatter plot and Table 1 provides some details. The LTMF (low percentage of trucks and medium flow) cluster (red circles) has a low \% of trucks (less than about $7 \%$ ) and up to a medium flow (less than about $1400 \mathrm{veh} /$ hour). The LTHF (low percentage of trucks and high flow) cluster (lilac stars) has a low \% trucks but a high flow. Due to the low \% trucks, neither the LTMF nor the LTHF clusters are critical for bridge traffic loading. The HTLF (high percentage of trucks and low flow) cluster (green squares) has a high \% trucks (from about $7 \%$ to $25 \%$ ) but low flow (below $700 \mathrm{veh} /$ hour). Because of the low flow, the inter-vehicle gaps will be large so, despite the high \% of trucks, this cluster will only be important for bridge traffic loading in the event of non-recurrent congestion (e.g., accident) that causes the gaps to be reduced. Finally, the MTHF (medium percentage of trucks and high flow) cluster (navy crosses) has a medium \% of trucks (from about 3\% to $16 \%$ ) and a high flow (about 700 to $3500 \mathrm{veh} / \mathrm{h}$ ). Because both the flow and the \% trucks can be relatively high, the number of trucks on the bridge and therefore the loading, can be significant.

The histograms of Figure 6 show the frequency of vehicles by hour of day for each cluster. The LTMF cluster can be seen to be strongly influenced by evening traffic, from about 19:00 to 21:00. In contrast, the LTHF cluster is mostly concentrated in the afternoon and evening rush hour. Most of the HTLF cluster comes from early morning traffic. Finally, the MTHF cluster is distributed through most of the day.

Table 1. Clusters features.

\begin{tabular}{|c|c|c|c|c|c|c|}
\hline $\begin{array}{l}\text { Cluster } \\
\text { No. }\end{array}$ & Name & $\begin{array}{l}\text { Symbols in } \\
\text { Figure } 5\end{array}$ & $\begin{array}{c}\text { Traffic } \\
\text { Characteristics }\end{array}$ & $\begin{array}{c}\text { Mean } \\
\text { Percentage } \\
\text { of Trucks }\end{array}$ & $\begin{array}{l}\text { No. of } \\
\text { Hours }\end{array}$ & No. of Days \\
\hline 1 & LTMF & Red circles & $\begin{array}{l}\text { Low } \% \text { trucks \& up } \\
\text { to medium flow }\end{array}$ & $3.6 \%$ & 478 & 130 \\
\hline 2 & LTHF & Lilac stars & $\begin{array}{c}\text { Low } \% \text { trucks \& up } \\
\text { to high flow }\end{array}$ & $2.3 \%$ & 443 & 116 \\
\hline 3 & HTLF & $\begin{array}{c}\text { Green } \\
\text { squares }\end{array}$ & $\begin{array}{c}\text { High } \% \text { trucks \& } \\
\text { low flow }\end{array}$ & $15.6 \%$ & 146 & 113 \\
\hline 4 & MTHF & $\begin{array}{l}\text { Navy blue } \\
\text { crosses }\end{array}$ & $\begin{array}{l}\text { Up to medium \% } \\
\text { trucks \& up to } \\
\text { high flow }\end{array}$ & $7.1 \%$ & 940 & 127 \\
\hline
\end{tabular}



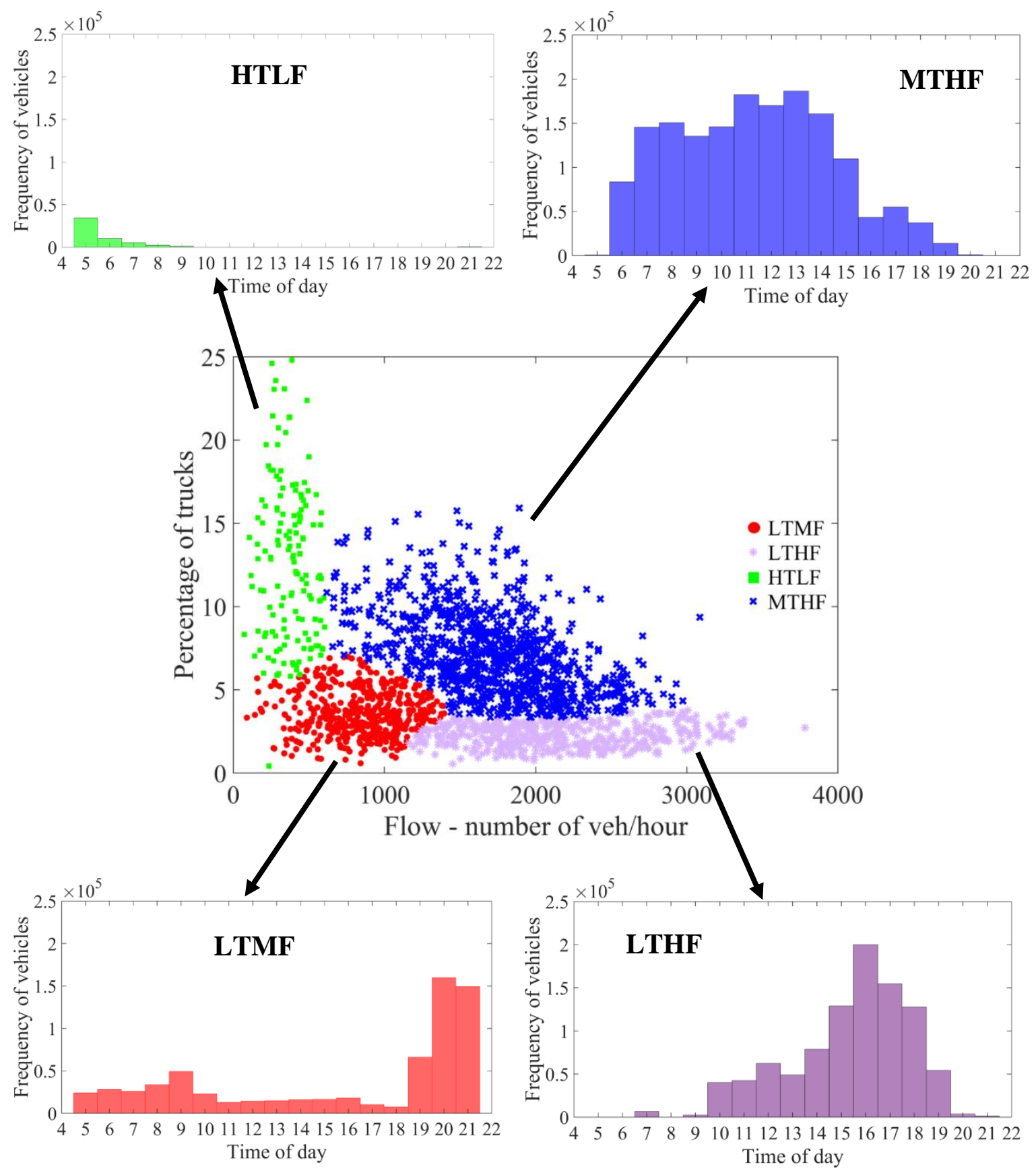

Figure 6. Data illustrated as four different clusters.

\section{Critical Cluster for Traffic Loading}

The load effect of cable axial force is calculated and plotted on probability paper for comparison of the four clusters. One thousand snapshots of traffic are extracted from each cluster. Each snapshot represents the full length of the FRB $(1813 \mathrm{~m})$ covered by regular traffic extracted from images. This is done by stitching together traffic data from images at the camera location, which covers about $50 \mathrm{~m}$, and it is acknowledged that it does not take account of the difference between one large image covering the full bridge length and a series of images taken at sequential points in time. As explained in Section 2.1, the weights are inferred from vehicle lengths. An influence line for cable force is derived from a detailed finite element model-Figure 7-and the axial force in the main cable on the West side is calculated for each snapshot. Southbound traffic, i.e., traffic on the East carriageway, is not considered. It is assumed that the West cable will carry a small part of that load but that this will be balanced by 
the East cable carrying a small part of the West carriageway load. It is assumed that extreme loading on the two carriageways is unlikely to occur at the same time and that, while this is a potential source of inaccuracy, it is not expected to have a big influence on the findings.

The cable forces are plotted on Gumbel probability paper [50] in Figure 8. Even though there is no theoretical basis for such an assumption, the data fits well to a Gumbel distribution [51] as evidenced by the approximate linearity of the data in each cluster. It can be seen that the HTLF cluster is to the left of the other three, despite a high \% of trucks, evidently because of the low flows involved. The LTMF and LTHF clusters are more prominent, with higher flows but low percentages of trucks. The dominant cluster is MTHF, significantly right of all others, as it has quite high flows and a medium percentage of trucks. These trends are accentuated by the relative frequencies of the clusters. As can be seen in Table 1, there are considerably fewer hours of data for the HTLF cluster data (reducing its probability for a given return period) and considerably more hours of MTHF data (increasing its probability). Given its dominance over the other clusters, the following sections relate to the MTHF cluster only.

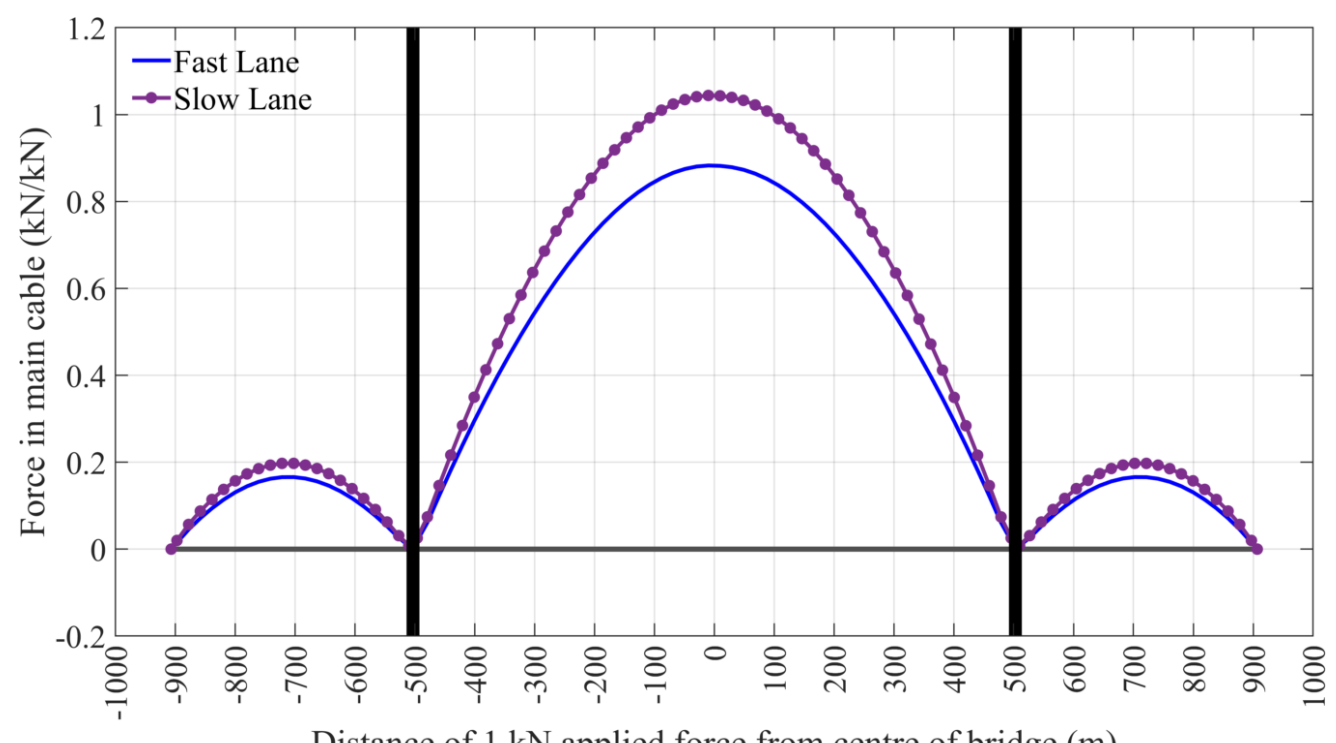

Distance of $1 \mathrm{kN}$ applied force from centre of bridge $(\mathrm{m})$

Figure 7. Influence lines for axial force in the main cable on the West side (northbound lanes).

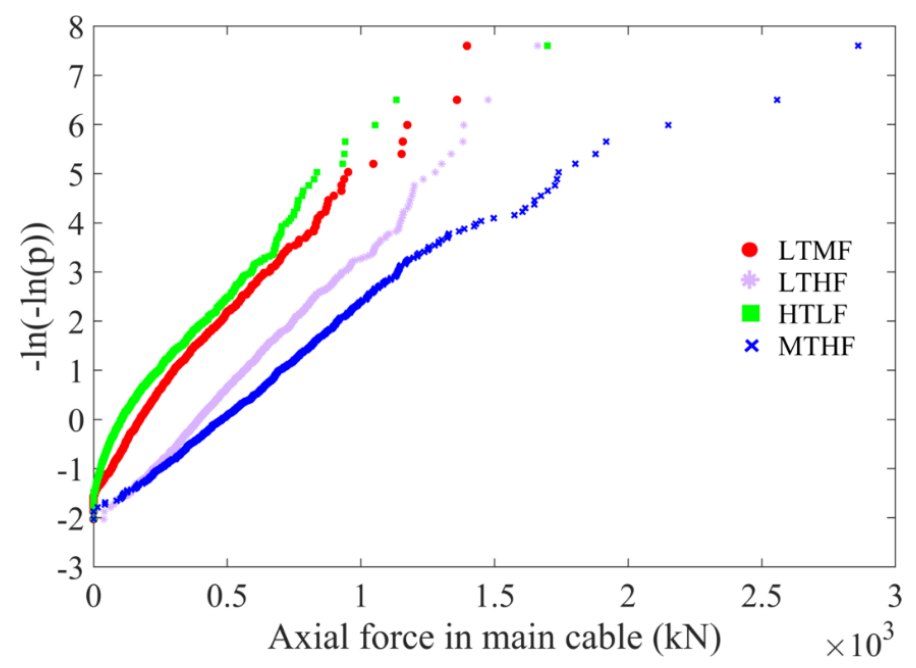

Figure 8. Probability paper plots of axial force in main cable for low trucks/medium flow (LTMF), low trucks/high flow (LTHF), high trucks/low flow (HTLF) and medium trucks/high flow (MTHF). 


\subsection{Influence of Bridge Length}

The calculated influence lines for the FRB are adjusted to provide estimates of similar influence lines for bridges with different spans. The FRB is $1813 \mathrm{~m}$ long with a main span of $1006 \mathrm{~m}$. For other spans, it is assumed that the relative dimensions of the towers/spans and the slopes of the suspension cables will be similar. It follows that the influence line ordinates, cable force due to unit applied point load, will be of similar magnitude to the FRB. The total lengths of the notional bridges are scaled to keep the same ratio of main- to side-span length. Based on these assumptions, Table 2 shows the total and main-span lengths for five notional bridges.

Table 2. Notional bridges lengths.

\begin{tabular}{ccc}
\hline Bridge No. & Main Span (m) & Total Length (m) \\
\hline $\mathbf{1}$ & $\mathbf{8 0 0}$ & 1441 \\
\hline 2 (FRB) & 1006 & 1813 \\
\hline 3 & 1200 & 2174 \\
\hline 4 & 1400 & 2524 \\
\hline 5 & 1600 & 2897 \\
\hline
\end{tabular}

An hour of data is generated as a very long train of vehicles that travels across the bridge. The load effect is recalculated as the train moves in increments of $10 \mathrm{~m}$ until the last vehicle in the train reaches the bridge. For the critical MTHF cluster, the maximum load effect per hour is identified for each of the $940 \mathrm{~h}$ of data (see Table 1). The process is repeated to find the maximum load effect per day for each of the 127 days of data. Figure 9 shows the probability paper plots for maximum-per-hour and maximum-per-day load effects on Gumbel probability paper. It is a matter of Engineering judgement as to which is the more effective way to estimate extreme values; it is essentially a trade-off between many points of less reliability or fewer points of greater reliability. As would be expected, the load effects increase with increasing bridge span, but the trends are consistent. The intensities of truck loading associated with these load effects have been calculated, i.e., the total combined length of truck as a percentage of total lane length. There is a significant trend of decreasing intensity as the span increases [52].

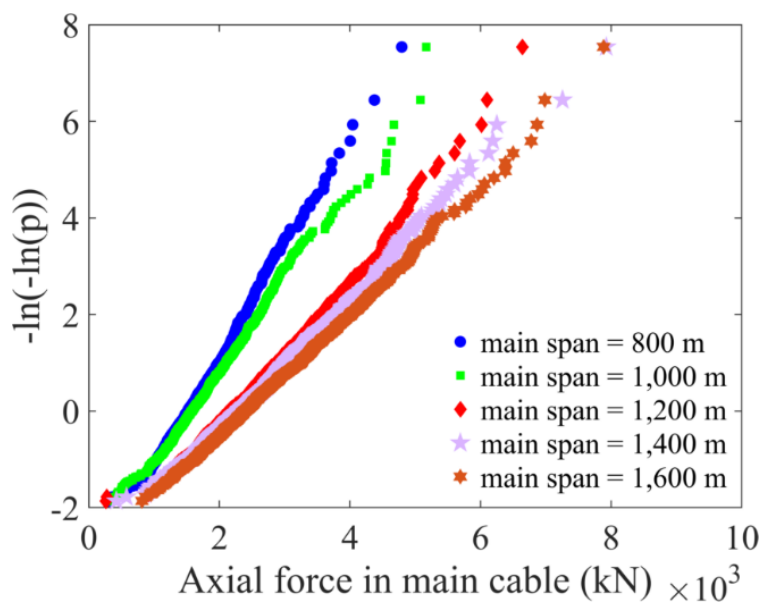

(a)

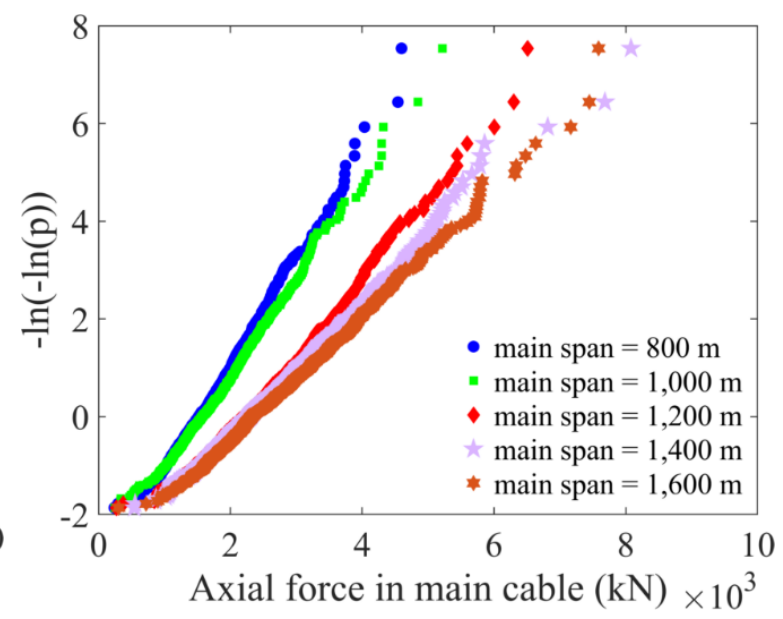

(b)

Figure 9. Probability paper plots of cable force for medium trucks/high flow (MTHF) for five bridge lengths: (a) maximum-per-hour; (b) maximum-per-day. 
Both the maximum-per-hour and maximum-per-day plots of Figure 9 are reasonably linear for all spans, confirming good fits to the Gumbel distribution. The maximum-per-day data is extrapolated to find characteristic maximum values. As data was collected 7 days per week, 1000-year and 75-year return periods correspond to probabilities of non-exceedance of $1-1 /(365 \times 1000)=0.999997$ (Eurocode) and $1-1 /(365 \times 75)=0.99996($ AASHTO $)$ respectively. Figure 10 shows the resulting characteristic maximum axial forces in the main cable values for both the 75- and 1000-year return periods for each bridge length. As expected, there is an increase in the characteristic values with span for both return periods. For example, as the span increases by $100 \%$, the 75 -year cable force increases by $65 \%$.

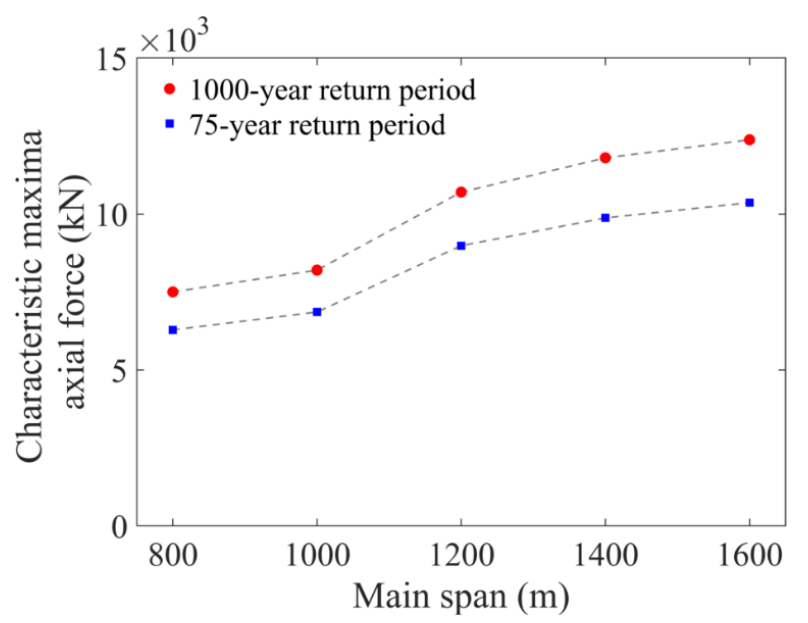

Figure 10. Characteristic maxima axial force for all five bridge lengths.

\subsection{Critical Loading Conditions}

Figure 11 presents a scatter plot for the critical MTHF cluster with contours showing the numbers of trucks per hour. The top $10 \%$ of the maximum-per-day axial forces in the main cable for the FRB are shown as pink pentagrams. It can be seen that, while there are exceptions, most of the more critical loading events come from hours when there are a greater number of trucks. Of the $14 \mathrm{~h}$ in the top 10\%, 12 of them are from the 13:00 time interval. It is concluded that, outside of exceptional events (accidents) for which there is insufficient data, the critical loading events happen when there is a medium percentage of trucks in a medium or high-flow situation. Within this cluster, the critical events are most likely to occur when there are greater numbers of trucks per hour. For this bridge, this tends to happen around 13:00.

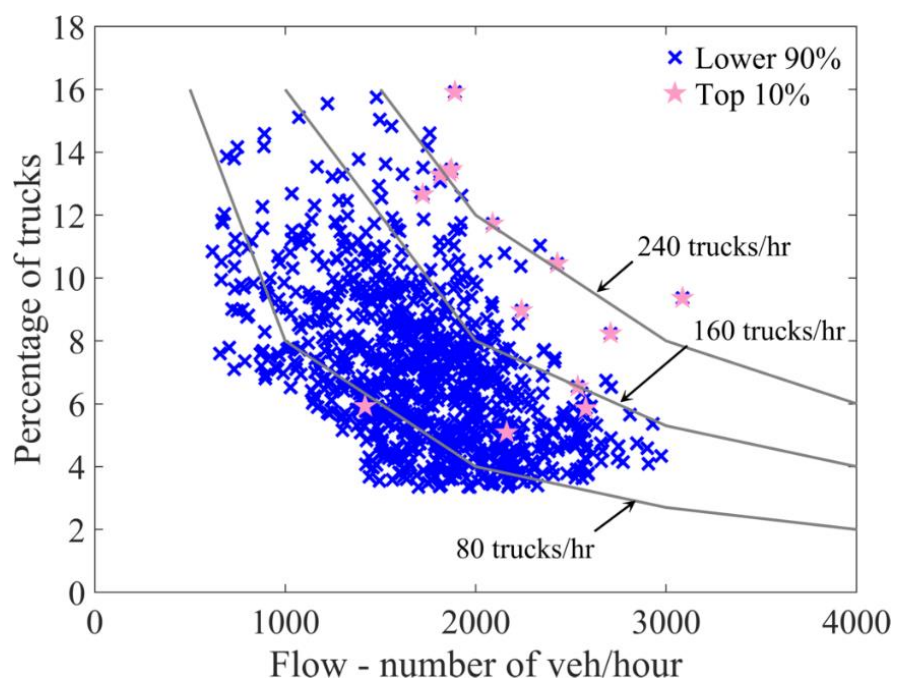

Figure 11. Scatter plot of MTHF cluster with contours showing number of trucks per hour. 
Exceptional or non-recurrent events such as accidents, can cause a very small number of fully jammed conditions per year. In five months of data collection on the FRB, two fully jammed situation were noted at the camera location. In a fully jammed situation, the inter-vehicle gaps implicit in vehicle flows are no longer relevant and the cluster where the percentage of trucks is highest, is assumed to govern. While it does not allow for lane changing, the gaps in the HTLF data are collapsed here to $1.5 \mathrm{~m}$ constant inter-vehicle distance [2] to simulate congestion. The weights of the vehicles are inferred as before. Axial force in the main cable on the West side is calculated using the available influence lines. The results obtained from 1000 jammed snapshots are presented on a Gumbel probability paper plot in Figure 12a. The top 35\% of the upper tail is reasonably linear and, therefore, follows a Gumbel distribution [50]. If such an accident were assumed to occur just once in ten years, then a 75-year return period would correspond to a non-recurrence probability of 1.94, giving a characteristic cable force $8505 \mathrm{kN}$. This value is equivalent to the 75-year characteristic maximum cable force due to regular traffic (MTHF) as illustrated in Figure 11 and in Figure 12b. Accidents are typically more frequent than this on the Forth Road Bridge, but it should be borne in mind that the snapshot assumes both lanes of the $1.8 \mathrm{~km}$ carriageway to be completely filled with congested traffic.

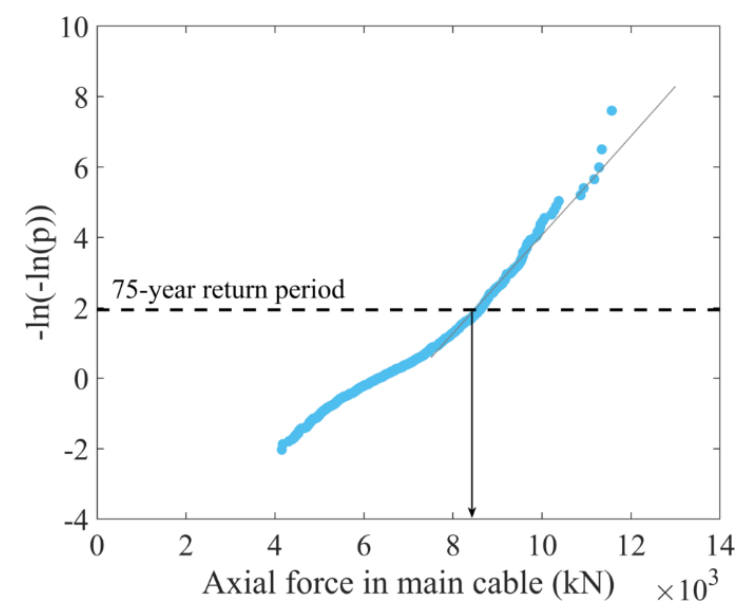

(a)

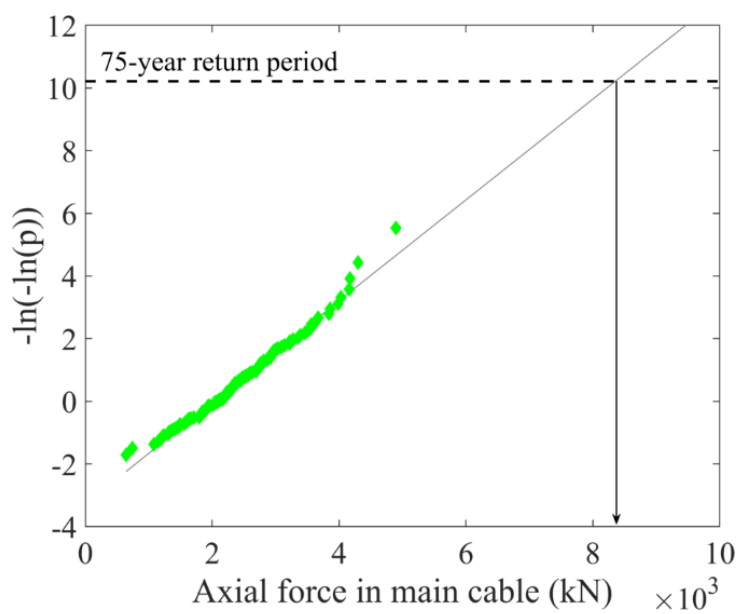

(b)

Figure 12. Comparison of cable forces for jammed and regular traffic: (a) Non-recurrent (jammed) from HTLF cluster; (b) Maximum-per-day cable forces due to regular traffic from MTHF cluster.

\section{Conclusions}

This paper investigates the nature of recurrent congested traffic loading, using the example of the Forth Road Bridge (FRB). Vehicle lengths and their location on the bridge are measured in more than two thousand hours of video data using standard image processing approaches. Monte Carlo sampling is applied to infer typical gross vehicle weight from length using an available WIM database of free-flowing traffic. This provides a unique combination of vehicle weight, gap and car/truck mix data for various stages of congestion. A dendrogram is used to identify the traffic with similar characteristics and to structure the data in a hierarchy. In accordance with the hierarchical classification, the traffic is sorted in four clusters on the basis of flow (vehicles per hour) and truck percentage. Histograms are used to compare cluster distributions throughout the day. They reveal that the cluster with a medium percentage of trucks and high flow (MTHF), which includes almost half of the video data $(940 \mathrm{~h})$, is spread over most of the $24 \mathrm{~h}$. Axial force in the main cable is calculated and plotted on a Gumbel probability paper plot to investigate the clusters' criticality. The MTHF cluster appears to be the dominant traffic condition for the FRB. For this cluster, maximum per hour and maximum per day load effect of cable axial force are calculated and compared for five notional bridges. The outcomes of this comparison show consistent trends for load effect of cable axial force. The site-specific characteristic 
maximum cable force determined for 75-year and 1000-year return periods increase slightly with increasing bridge span. In comparing 'regular' recorded traffic with an accident situation generated by collapsing all gaps to minimum values, the risk from regular traffic, as measured every day, is found to be similar to that due to a major accident.

Author Contributions: Conceptualization, E.A.M. and E.J.O.; Data curation, E.A.M.; Funding acquisition, E.J.O.; Investigation, E.A.M.; Methodology, E.A.M. and E.J.O.; Resources, R.M. and E.A.; Supervision, E.J.O., A.M., M.L. and F.N.C.; Writing-original draft, E.A.M.; Writing-review and editing, E.J.O. All authors have read and agreed to the published version of the manuscript.

Funding: This research was funded by Science Foundation Ireland under US/Ireland programme, grant 14/US/I3033.

Acknowledgments: The authors gratefully acknowledge Science Foundation Ireland for supporting this research under US/Ireland programme grant 14/US/I3033.

Conflicts of Interest: The authors declare no conflict of interest. The funder had no role in the design of the study; in the collection, analyses, or interpretation of data; in the writing of the manuscript, or in the decision to publish the results.

\section{References}

1. Buckland, P.G.; McBryde, J.P.; Navin, F.P.; Zidek, J.V. Traffic loading of long span bridges. Bridge Eng. 1978, 2, 146-154.

2. Buckland, P.G.; Navin, F.P.D.; Zidek, J.V.; McBryde, J.P. Proposed vehicle loading of long-span bridges. J. Struct. Div. 1980, 106, 915-932.

3. Al-Kaisy, A.F.; Hall, F.L.; Reisman, E.S. Developing passenger car equivalents for heavy vehicles on freeways during queue discharge flow. Transp. Res. Part A Policy Pr. 2002, 36, 725-742. [CrossRef]

4. Ivy, R.J.; Lin, T.Y.; Mitchell, S.; Raab, N.C.; Richey, V.J.; Scheffey, C.F. Live Loading for Long-Span Highway Bridges; American Society of Civil Engineers Transactions: Washington, DC, USA, 1954.

5. Getachew, A. Traffic Load Effects on Bridges, Statistical Analysis of Collected and Monte Carlo Simulated Vehicle Data. Ph.D. Thesis, Royal Institute of Technology, Stockholm, Sweden, 2003.

6. Caprani, C.C.; OBrien, E.J. The governing form of traffic for highway bridge loading. In Proceedings of the 4th Symposium on Bridge and Infrastructure Research in Ireland, Galway, Ireland, 4-5 December 2008; pp. 53-60.

7. Moses, F. Weigh-in-Motion System Using Instrumented Bridges. Transp. Eng. J. ASCE 1979, 105, 233-249.

8. OBrien, E.J.; Žnidarič, A.; Ojio, T. Bridge weigh-in-motion-Latest developments and applications world wide. In Proceedings of the International Conference on Heavy Vehicles, Paris, France, 19-22 May 2008; pp. 19-22.

9. Obrien, E.J.; Lipari, A.; Caprani, C. Micro-simulation of single-lane traffic to identify critical loading conditions for long-span bridges. Eng. Struct. 2015, 94, 137-148. [CrossRef]

10. Bailey, S.F. Basic Principles and Load Models for the Structural Safety Evaluation of Existing Road Bridges; Civil Engineering, Ecole Polytechnique Fédérale de Lausanne: Lausanne, Switzerland, 1996.

11. Bruls, A.; Croce, P.; Sanpaolesi, L.; Sedlacek, G. ENV 1991-Part 3: Traffic Loads on Bridges Calibration of Road Load Models for Road Bridges, IABSE REPORTS: Basis of Design and Actions on Structures; Background and application of Eurocode 1; IABSE: Delft, The Netherlands, 1996; pp. 439-454.

12. Buckland, P.G. North-American and British Long-Span Bridge Loads. J. Struct. Eng. 1991, 117, 2972-2987. [CrossRef]

13. Hayrapetova, A.; O'Connor, A.; Obrien, E.J. Traffic Load Models for Long Span Bridges, Bridge Maintenance, Safety, Management, Resilience \& Sustainability; Taylor \& Francis Group: Stresa, Italy, 2012; pp. 2589-2596.

14. O'Brien, E.; Hayrapetova, A.; Walsh, C. The use of micro-simulation for congested traffic load modeling of medium- and long-span bridges. Struct. Infrastruct. Eng. 2012, 8, 269-276. [CrossRef]

15. Vrouwenvelder, A.C.W.M.; Waarts, P.H. Traffic Loads on Bridges. Struct. Eng. Int. 1993, 3, 169-177. [CrossRef]

16. Ditlevsen, O. Traffic Loads on Large Bridges Modeled as White-Noise Fields. J. Eng. Mech. 1994, 120, 681-694. [CrossRef]

17. Paeglitis, A.; Freimanis, A. Comparision of constant-span and influence line methods for long-span bridge load calculations. Balt. J. Road Bridg. Eng. 2016, 11, 84-91. [CrossRef] 
18. Lutomirska, M.; Nowak, A. Live Load Models for Long Span Bridges; Civil Engineering, University of Nebraska: Lincoln, NE, USA, 2009.

19. Nowak, A.; Lutomirska, M.; Ibrahim, F.S. The development of live load for long span bridges. Bridg. Struct. 2010, 6, 73-79. [CrossRef]

20. Nowak, A.; Hong, Y. Bridge Live-Load Models. J. Struct. Eng. 1991, 117, 2757-2767. [CrossRef]

21. Caprani, C. Using microsimulation to estimate highway bridge traffic load. In Proceedings of the 5th International Conference on Bridge Maintenance, Safety, Management, Resilience and Sustainability, Philadelphia, PA, USA, 11-15 July 2010.

22. Enright, B.; Carey, C.; Caprani, C. Microsimulation Evaluation of Eurocode Load Model for American Long-Span Bridges. J. Bridge Eng. 2013, 18, 1252-1260. [CrossRef]

23. Treiber, M.; Hennecke, A.; Helbing, D. Microscopic simulation of congested traffic. In Traffic and Granular Flow'99; Helbing, D., Herrmann, H.J., Schreckenberg, M., Wolf, D.E., Eds.; Springer: Berlin/Heidelberg, Germany, 2000; pp. 365-376.

24. Caprani, C.; Obrien, E.J.; Lipari, A. Long-span bridge traffic loading based on multi-lane traffic micro-simulation. Eng. Struct. 2016, 115, 207-219. [CrossRef]

25. Hidas, P.; Wagner, P. Review of data collection methods for microscopic traffic simulation. In Proceedings of the 10th World Conference on Transport Research Istanbul, Istanbul, Turkey, 4-8 July 2004.

26. Treiterer, J.; Myers, J. The hysteresis phenomenon in traffic flow. In Proceedings of the 6th International Symposium on Transportation and Traffic Theory, Sydney, Australia, 26-28 August 1974; pp. 13-38.

27. Wang, L.; Chen, F.; Yin, H. Detecting and tracking vehicles in traffic by unmanned aerial vehicles. Autom. Constr. 2016, 72, 294-308. [CrossRef]

28. Xu, J.; Wang, G.; Sun, F. A novel method for detecting and tracking vehicles in traffic-image sequence. In Proceedings of the Fifth International Conference on Digital Image Processing (ICDIP 2013), Beijing, China, 21-22 April 2013.

29. Zhang, G.; Avery, R.P.; Wang, Y. Video-Based Vehicle Detection and Classification System for Real-Time Traffic Data Collection Using Uncalibrated Video Cameras. Transp. Res. Rec. J. Transp. Res. Board 2007, 1993, 138-147. [CrossRef]

30. Morris, B.; Trivedi, M. Robust classification and tracking of vehicles in traffic video streams. In Proceedings of the 2006 IEEE Intelligent Transportation Systems Conference, Toronto, ON, Canada, 17-20 September 2006; pp. 1078-1083.

31. Coifman, B.; Beymer, D.; Mclauchlan, P.; Malik, J. A real-time computer vision system for vehicle tracking and traffic surveillance. Transp. Res. Part C Emerg. Technol. 1998, 6, 271-288. [CrossRef]

32. Reinartz, P.; Lachaise, M.; Schmeer, E.; Krauss, T.; Runge, H. Traffic monitoring with serial images from airborne cameras. ISPRS J. Photogramm. Remote. Sens. 2006, 61, 149-158. [CrossRef]

33. Farnebäck, G.; Nordberg, K. Motion detection in the WITAS project. In Proceedings of the Swedish Symposium on Image Analysis (SSBA), Lund, Sweden, 13-14 March 2008; pp. 99-102.

34. Fu, T.; Zangenehpour, S.; St-Aubin, P.; Fu, L.; Miranda-Moreno, L.F. Using microscopic video data measures for driver behavior analysis during adverse winter weather: Opportunities and challenges. J. Mod. Transp. 2015, 23, 81-92. [CrossRef]

35. Ricketts, N.; Page, J. Traffic Data for Highway Bridge Loading; TRL REPORT 251; Transport Research Laboratory: Crowthorne, UK, 1997.

36. Micu, E.A.; Obrien, E.J.; Malekjafarian, A.; Quilligan, M. Estimation of Extreme Load Effects on Long-Span Bridges Using Traffic Image Data. Balt. J. Road Bridge Eng. 2018, 13, 429-446. [CrossRef]

37. Micu, E.A.; McKinstray, R.; Angus, E.; OBrien, E.J.; Malekjafarian, A.; Lydon, M. Estimation of traffic load effects on Forth Road Bridge using camera measurements. In Civil Engineering Research in Ireland 2018 (CERI2018); Pakrashi, V., Keenahan, J., Eds.; Civil Engineering Research Association of Ireland: Dublin, Ireland, 2018; pp. 79-83.

38. Bradley, D.; Roth, G. Adaptive Thresholding using the Integral Image. J. Graph. Tools 2007, 12, 13-21. [CrossRef]

39. Vincent, L. Morphological grayscale reconstruction in image analysis: Applications and efficient algorithms. IEEE Trans. Image Process. 1993, 2, 176-201. [CrossRef] [PubMed] 
40. Micu, E.A.; Malekjafarian, A.; Obrien, E.J.; Quilligan, M.; McKinstray, R.; Angus, E.; Lydon, M.; Catbas, F.N. Evaluation of the extreme traffic load effects on the Forth Road Bridge using image analysis of traffic data. Adv. Eng. Softw. 2019, 137, 102711. [CrossRef]

41. McKinstray, R.; Angus, E.; OBrien, E.J.; Micu, E.A.; Malekjafarian, A.; Lydon, M. Image data for bridge traffic load monitoring on Forth Road Bridge. In Proceedings of the 17th European Bridge Conference, Edinburgh, Scotland, 15-17 May 2018.

42. Enright, B. Simulation of Traffic Loading on Highway Bridges. Ph.D. Thesis, University College Dublin, Dublin, Ireland, 2010.

43. Jones, M.C.; Kappenman, R.F. On a Class of Kernel Density Estimate Bandwidth Selectors. Scand. J. Stat. 1992, 19, 337-349.

44. Gan, G.; Ma, C.; Wu, J. Data Clustering: Theory, Algorithms, and Applications; SIAM: Philadelphia, PA, USA, 2007.

45. Kaufman, L.; Rousseeuw, P.J. Finding Groups in Data: An Introduction to Cluster Analysis; John Wiley \& Sons: New York, NY, USA, 2009; Volume 344.

46. McLachlan, G.J.; Peel, D. Finite Mixture Models; John Wiley \& Sons, Inc.: New York, NY, USA, 2000.

47. Everitt, B.S.; Landau, S.; Leese, M.; Stahl, D. Cluster Analysis, 5th ed.; John Wiley \& Sons, Inc.: New York, NY, USA, 2011.

48. Biernacki, C.; Celeux, G.; Govaert, G. Choosing starting values for the EM algorithm for getting the highest likelihood in multivariate Gaussian mixture models. Comput. Stat. Data Anal. 2003, 41, 561-575. [CrossRef]

49. Hansen, P.; Jaumard, B.; Simeone, B. Espaliers: A generalization of dendrograms. J. Classif. 1996, 13, $107-127$. [CrossRef]

50. Castillo, E. Extreme Value Theory in Engineering; Academic Press: New York, NY, USA, 1988.

51. Benjamin, J.R.; Cornell, C.A. Probability, Statistics and Decision for Civil Engineers; McGraw-Hill: New York, NY, USA, 1970.

52. Micu, E.A. Using Traffic Image Data for Load Monitoring on Long-Span Bridges; University College Dublin: Dublin, Ireland, 2019.

(C) 2020 by the authors. Licensee MDPI, Basel, Switzerland. This article is an open access article distributed under the terms and conditions of the Creative Commons Attribution (CC BY) license (http://creativecommons.org/licenses/by/4.0/). 